\title{
Easy Access to 2-Fluoro- and 2-lodo-2H-azirines via the Halex Reaction
}

\author{
Anastasiya V. Agafonova \\ Ilia A. Smetanin \\ Nikolai V. Rostovskii \\ Alexander F. Khlebnikov \\ Mikhail S. Novikov* \\ Saint Petersburg State University, Institute of Chemistry, \\ 7/9 Universitetskaya nab., St. Petersburg, 199034, Russia \\ m.novikov@spbu.ru
}

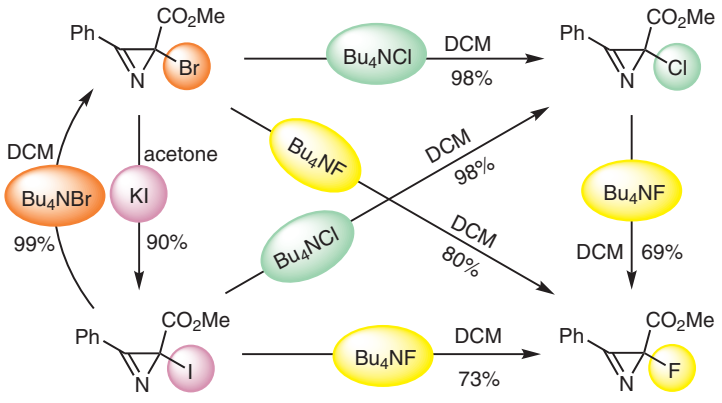

ing probes. Such reactions, in particular, include nucleophilic substitution reactions, which can use commercially available isotopically labeled alkali metal fluorides and iodides as reagents.

One of the challenges of this chemistry is the introduction of halogen atoms into labile structures, which are easily destroyed by standard halogenating agents. It is particularly true for $2 \mathrm{H}$-azirines, the strain energy of the threemembered ring of which makes them attractive building blocks for the synthesis of four-, five-, and six-membered heterocycles. ${ }^{8}$ Examples of the use of 2-bromoazirines for the preparation of the azete and oxazoline derivatives, proceeding with the preservation of the halogen atom in the product have also been reported. ${ }^{9} 2$-Fluoro- and 2-iodoazirines are much less accessible compounds, and their chemistry is practically unexplored. Besides, 2-iodo- $2 \mathrm{H}$-azirines are much more reactive in substitution reactions than 2-bromoazirines, and there are cases when less accessible iodides cannot be replaced by more accessible bromides. ${ }^{10}$

The known methods for the synthesis of these compounds are based on two strategies. In the framework of the first strategy, the 2-haloazirine ring is formed through the cyclization of halogenated open-chain precursor, for example, through thermal cyclization of a 2-halovinylazide. Some 2-iodo- and 2-fluorovinylazides can be obtained by dehydrohalogenation of 1-azido-2,2-dihalo derivatives ${ }^{11}$ or from $\alpha$-oxophosphonium ylides and $\mathrm{N}$-iodosuccinimide in the presence of azidotrimethylsilane (Scheme 1, reaction 1). ${ }^{12}$ The reaction of $\beta$-carbonyl-substituted enamines with $\mathrm{PhIF}_{2}$ generated in situ from PhIO and $\mathrm{Et}_{3} \mathrm{~N} \cdot 3 \mathrm{HF}$ was used for the preparation of 2-fluoroazirines, containing a carbonyl substituent at the C2 atom (reaction 4)..$^{13}$ Another strategy for the synthesis of 2-fluoro- and 2-iodoazirine-2carbonyls is based on the metal-catalyzed ring contraction of 4-fluoro- and 4-iodoisoxazoles (reactions 2 and 3). ${ }^{14,15}$ Unfortunately, the methods noted above require either po- 
tentially explosive compounds or expensive reagents. For the preparation of starting materials such as 4-fluoro- and 4-iodoisoxazoles, butyllithium is required. All of this calls into question the scalability of such protocols and stimulates the search for new methods for the preparation of 2-fluoro- and 2-iodoazirines.

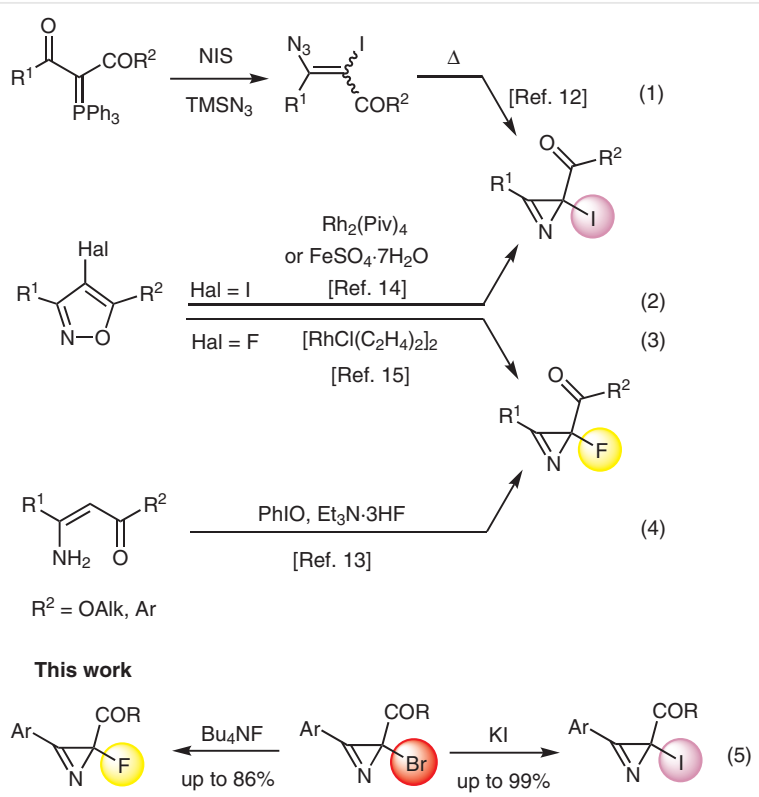

Scheme 1 Synthesis of 2-halo-2H-azirine-2-carbonyl compounds

One powerful method for the introduction of halogen atoms into a cyclic molecule is the halogen exchange (Halex) reaction. A transition-metal-free version of this method is widely used for the preparation of haloarenes ${ }^{16}$ and, to a lesser extent, for the synthesis of haloheteroarenes, such as fluorotriazoles ${ }^{17}$ and fluoropyridines. ${ }^{18}$ In this work, a new method for the synthesis of 2-fluoro- and 2-iodoazirine-2carbonyls based on the halogen exchange reaction is developed (Scheme 1, reaction 5). This method uses easily available 2-bromo-2H-azirine-2-carboxylic acid derivatives as starting materials and inexpensive and safe reagents for the introduction of fluorine and iodine into the azirine ring.

Recently, we have shown that a bromine atom in 2-bromo-2H-azirine-2-carboxylates can be easily substituted with acyloxy-, alkoxy-, alkenyloxy- ${ }^{10}$ and azolyl ${ }^{19}$ substituents under mild conditions. These transformations take place under the action of the corresponding $\mathrm{O}$ - and $\mathrm{N}$-nucleophiles via a cascade $\mathrm{S}_{\mathrm{N}} 2^{\prime}-\mathrm{S}_{\mathrm{N}} 2^{\prime}$ mechanism, which imposes high requirements on both the nucleophilicity and the nucleofugality of the reagent. Thus, aliphatic and aromatic carboxylic acids react with methyl 2-bromo-3-phenyl-2H-azirine-2-carboxylate (1a) in the presence of $\mathrm{Et}_{3} \mathrm{~N}$ to afford 2-acyloxy derivatives in high yields, while with substantially more acidic trichloroacetic acid the reaction does not occur. ${ }^{10}$ As far as we know, there is no information in the literature on the use of halide ions as nucleophiles in such reactions.

We began our study with the search for optimal conditions for the preparation of fluoroazirine $\mathbf{2 a}$ via the halogen exchange reaction of 2-bromoazirine 1a. The latter was chosen because of its synthetic accessibility and high reactivity in the substitution reactions. ${ }^{10,19}$

It was found that $\mathbf{1 a}$ reacted with tetrabutylammonium fluoride hydrate (TBAF. $\mathrm{H}_{2} \mathrm{O}$ ) in toluene at ambient temperature to afford the target fluoride $\mathbf{2 a}$ in good yield (Table 1, entry 1). Screening of other sources of fluoride ion and solvents (entries 2-6) showed that the highest yield of fluoride 2a from bromide 1a was achieved using TBAF. $\mathrm{H}_{2} \mathrm{O}(1.3$ equiv) in dichloromethane (DCM) at room temperature (entry 2). These conditions were used in further experiments carried out to evaluate the substrate scope of the reaction.

Table 1 Optimization of Azirine 2a Synthesis

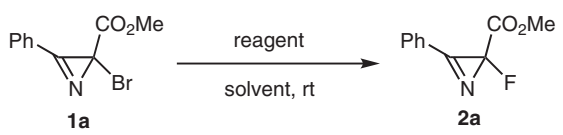

\begin{tabular}{lllll}
\hline Entry & Reagent (equiv) & Solvent & Time (h) & Yield of 2a (\%) \\
\hline 1 & TBAF $\cdot \mathrm{H}_{2} \mathrm{O}(1.3)$ & toluene & 4 & 76 \\
2 & $\mathrm{TBAF} \cdot \mathrm{H}_{2} \mathrm{O}(1.3)$ & $\mathrm{DCM}$ & 4 & 80 \\
3 & $\mathrm{KF}(1.5)$ & DMSO & 12 & 33 \\
4 & $\mathrm{CsF}(1.5)$ & DMSO & 12 & 45 \\
5 & $\mathrm{CoF}_{2}(1.5)$ & DMSO & 48 & trace \\
6 & $\mathrm{FeF}_{2}(1.5)$ & toluene & 12 & 0 \\
\hline
\end{tabular}

a Yield of isolated product.

Bromoazirines 1a-n required for the experiments were prepared from 5-(alkoxy/amino)isoxazoles 5a-n by bromination using $\mathrm{N}$-bromosuccinimide (NBS) followed by $\mathrm{FeSO}_{4}$ catalyzed isomerization of bromoisoxazoles 6a-n (Scheme 2). ${ }^{14 a}$ Isoxazoles 5 were synthesized from either 5chloroisoxazoles $\mathbf{3}$ or isoxazol-5-ones $\mathbf{4}^{14 \mathrm{~b}}$

The substitution reaction worked well with 2-bromo-3aryl-2H-azirine-2-carboxylic esters containing both electron-withdrawing (Scheme 3, compound 2e) and electrondonating (Scheme 3, compound $\mathbf{2 g}$ ) aryl group and afforded the fluoroazirines in good to excellent yields. The reaction was also insensitive to steric hindrance introduced by bulky ortho-substituted aryl groups that could restrict the approach of the nucleophile (Scheme 3, compounds $\mathbf{2 d , f , h}$ ). This simple protocol could also be applied to 2-bromo-3aryl-2H-azirine-2-carboxamides, but the yields of the fluoroazirine-2-carboxamides were significantly lower (Scheme 3, compounds $\mathbf{2 k}, \mathbf{1}$ ). To test the ability to scale-up the synthesis of fluoroazirines $\mathbf{2}$, a gram-scale reaction of bromoazirine 1a (2.2 g, $8.66 \mathrm{mmol})$ with TBAF $\cdot \mathrm{H}_{2} \mathrm{O}(3.14 \mathrm{~g}$, $11.26 \mathrm{mmol}$ ) was carried out to give fluoroazirine $\mathbf{2 a}$ in $80 \%$ (1.34 g) yield. 

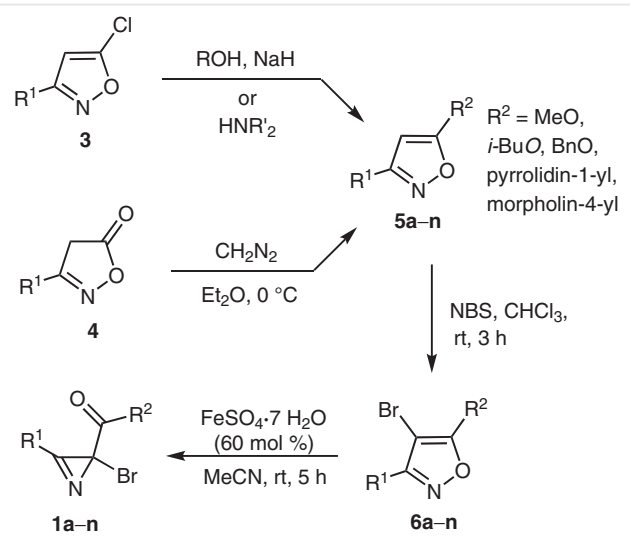

Scheme 2 Synthesis of bromoazirines $\mathbf{1 a - n}$
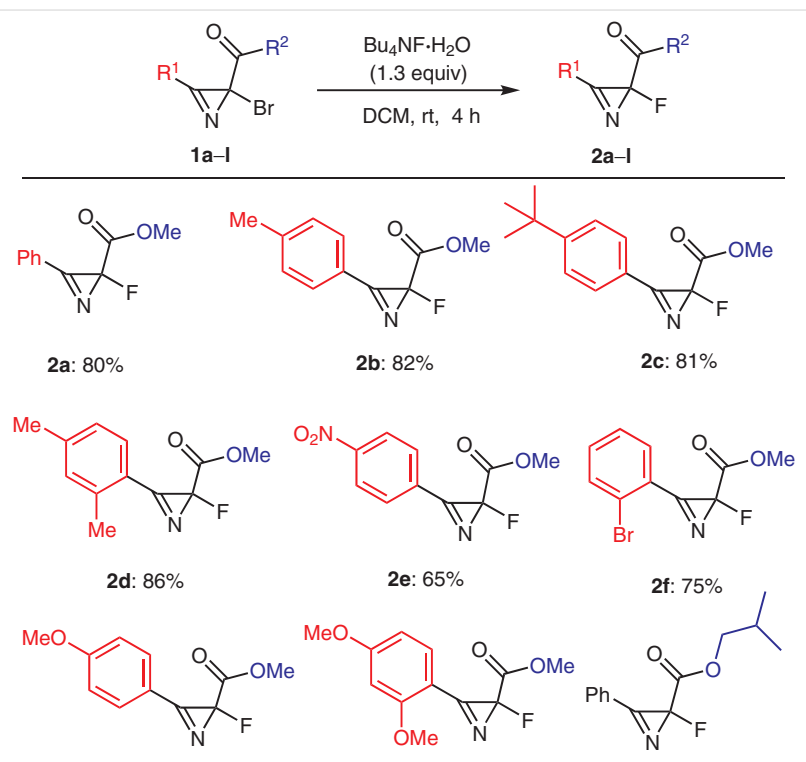

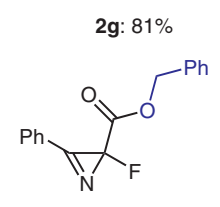

2j: $95 \%$

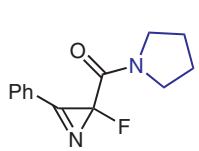

2k: $64 \%$

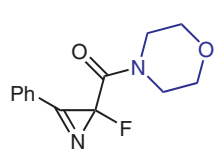

2I: $40 \%$
Scheme 3 Synthesis of fluoroazirines $\mathbf{2}$ from bromoazirines $\mathbf{1}$

It was also shown that the treatment of bromoazirine 1a with potassium iodide in acetone allowed the replacement of the bromine with iodine in high yield (Scheme 4, compound 7a). For the complete conversion of bromoazirines $\mathbf{1 a}, \mathbf{b}, \mathbf{e}, \mathbf{j}$, having no alkoxy groups in the aryl substituent, three equivalents of potassium iodide were required. When using tetrabutylammonium iodide (3 equiv) instead of potassium iodide, no reaction was observed. Unexpectedly, the conversion of alkoxy-substituted bromoazirines $\mathbf{1 m}, \mathbf{n}$ into iodoazirines 7d,e under the standard conditions was only 27\%; after this the reaction stopped. Iodoazirines 7d,e were obtained in $90 \%$ yield only when a 15 -fold excess of $\mathrm{KI}$ was used. This may be due to a significantly higher solubility of $\mathrm{KBr}$, compared with $\mathrm{KI}$ in solutions containing ether compounds, ${ }^{20}$ to which can be attributed compounds $\mathbf{1 m}, \mathbf{n}$ and 7d,e.

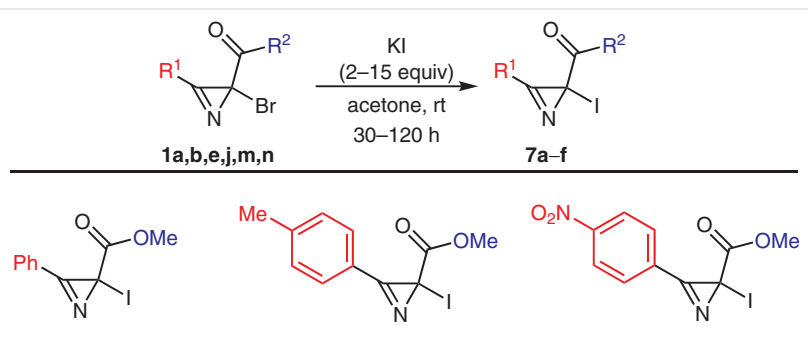

$$
\text { 7a: (92\%, from 1b) 7c: }(80 \% \text {, from 1e) }
$$

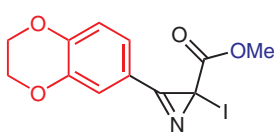

Scheme 4 Synthesis of iodoazirines 7 from bromoazirines 1

It was found that fluoroazirine $\mathbf{2 a}$ can be alternatively synthesized from iodoazirine 7a or chloroazirine 8a under the standard conditions (Scheme 5). The latter compound, in turn, can be obtained in practically quantitative yield by treating azirine 1a or 7a with tetrabutylammonium chloride in DCM. Unfortunately, the reverse transformation of chloroazirine 8a into bromoazirine 1a using tetrabutylammonium bromide ( 3 equiv) could not be carried out. This reaction does not occur at all. However, bromoazirine 1a was obtained by treating iodoazirine 7a with $\mathrm{Bu}_{4} \mathrm{NBr}$ (2 equiv) in DCM at room temperature in practically quantitative yield (Scheme 5).

Taking into account the results of the calculations and experimental data obtained for the halogen substitutions in 2-halo-2H-azirine-2-carboxylates with $\mathrm{O}$ - and $\mathrm{N}$-nucleophiles, ${ }^{10,19}$ we believe that the halogen exchange reactions described above also proceed via a cascade $S_{N} 2^{\prime}-S_{N} 2^{\prime}$ mechanism.

All the newly synthesized haloazirines $\mathbf{1 , 2}$, and 7 were characterized based of ${ }^{1} \mathrm{H},{ }^{13} \mathrm{C}$ NMR spectroscopic and HRMS data.

In summary, we have developed a simple synthesis of esters and dialkylamides of 2-(fluoro/iodo)- $2 \mathrm{H}$-azirine-2carboxylic acids from much more synthetically accessible 2-bromo-analogues by the halogen exchange (Halex) reaction. The method operates with inexpensive and safe reagents, TBAF and potassium iodide, and provides high product yields. For the synthesis of the 2-fluoro-substituted azirine from the corresponding bromides, a small excess of TBAF is sufficient. A threefold excess of KI in acetone is required to replace bromine with iodine in most 3-aryl-2- 


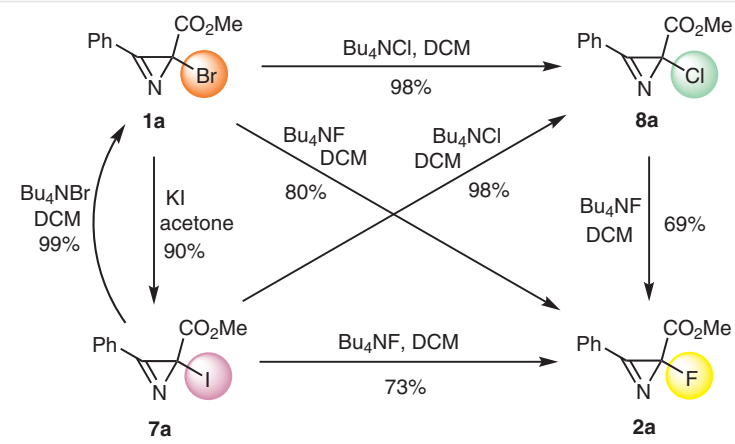

Scheme 5 Halex reactions of methyl 2-halo-3-phenylazirine-2-carboxylates

bromo-2H-azirine-2-carboxylates, with the exception of those that contain alkoxy groups in the aryl substituent. In the latter case, a 15 -fold excess of the reagent enables high yields of the iodoazirines to be achieved. Alternatively, 2-fluoro- $2 \mathrm{H}$-azirine-2-carboxylates can be prepared from 2-iodo- and 2-chloro-analogues. The latter compounds, in turn, can be obtained in practically quantitative yield by treating the 2-iodo- and 2-bromoazirines with $\mathrm{Bu}_{4} \mathrm{NCl}$.

Melting points were determined with a SMP30 melting point apparatus. ${ }^{1} \mathrm{H}(400 \mathrm{MHz})$ and ${ }^{13} \mathrm{C}(100 \mathrm{MHz})$ NMR spectra were recorded with a Bruker AVANCE 400 spectrometer in $\mathrm{CDCl}_{3}$. Chemical shifts $(\delta)$ are reported in ppm downfield from tetramethylsilane. Electrospray ionization (ESI), positive mode, mass spectra were measured with a Bruker MaXis mass spectrometer using acetonitrile for dilution of samples. Thin-layer chromatography (TLC) was conducted on aluminum sheets precoated with $\mathrm{SiO}_{2}$ ALUGRAM SIL G/UV254. Column chromatography was performed on Macherey-Nagel silica gel $60 \mathrm{M}$ (0.04-0.063 mm). All solvents were distilled and dried prior to use. Toluene was distilled and stored over sodium metal. Dichloromethane was washed with concentrated $\mathrm{H}_{2} \mathrm{SO}_{4}$ and water, then distilled from $\mathrm{P}_{2} \mathrm{O}_{5}$ and stored over anhydrous $\mathrm{K}_{2} \mathrm{CO}_{3}$. Acetone was distilled from anhydrous $\mathrm{CaCO}_{3}$.

Bromoazirines 1a,b,e,g,k,1, ${ }^{14}$ are known compounds and have full characterization data. They were synthesized according to the reported procedures that are illustrated in Scheme 2 .

\section{Synthesis of Bromoazirines 1c,f,h-j,m}

\section{Isoxazoles $5 f$ and 5i; General Procedure}

To a stirred suspension of $\mathrm{NaH}(60 \%$ in oil, $440 \mathrm{mg}, 11 \mathrm{mmol}$, prewashed with hexane) in anhydrous THF $(20 \mathrm{~mL})$, alcohol $(15 \mathrm{mmol})$ was added at ambient temperature and the reaction mixture was stirred for 0.5 h. 5-Chloro-3-arylisoxazole $3 \mathbf{a}\left(\mathrm{R}=2-\mathrm{BrC}_{6} \mathrm{H}_{4}\right)$ or $\mathbf{3 b}$ $(\mathrm{R}=\mathrm{Ph})^{21 \mathrm{a}}(5.6 \mathrm{mmol})$ was added and the mixture was heated at reflux for $1 \mathrm{~h}$. The mixture was cooled to ambient temperature, the reaction was quenched with water $(20 \mathrm{~mL})$ and the mixture was extracted with $\mathrm{CH}_{2} \mathrm{Cl}_{2}(3 \times 20 \mathrm{~mL})$. The combined organic layers were dried over $\mathrm{Na}_{2} \mathrm{SO}_{4}$ and concentrated under reduced pressure to give pure compound $\mathbf{5 f}$ or $\mathbf{5 i}$.

\section{3-(2-Bromophenyl)-5-methoxyisoxazole (5f)}

Yield: $1.31 \mathrm{~g}(92 \%)$ (from isoxazole 3a); colorless oil.

${ }^{1} \mathrm{H} \mathrm{NMR}\left(400 \mathrm{MHz}, \mathrm{CDCl}_{3}\right): \delta=7.72-7.66(\mathrm{~m}, 1 \mathrm{H}), 7.66-7.60(\mathrm{~m}, 1 \mathrm{H})$, 7.44-7.36 (m, $1 \mathrm{H})$, 7.36-7.29 (m, $1 \mathrm{H}), 5.67$ (s, $1 \mathrm{H}), 4.07$ (s, $3 \mathrm{H})$.

${ }^{13} \mathrm{C}$ NMR $\left(100 \mathrm{MHz}, \mathrm{CDCl}_{3}\right): \delta=173.6,164.3,133.6,131.1,130.0$, 130.9, 127.5, 122.1, 76.7, 58.8.

HRMS (ESI): $m / z[\mathrm{M}+\mathrm{Na}]^{+}$calcd for $\mathrm{C}_{10} \mathrm{H}_{8}{ }^{81} \mathrm{BrNNaO}_{2}{ }^{+}:$277.9610; found: 277.9606 .

\section{5-Isobutoxy-3-phenylisoxazole (5i)}

Yield: $1.20 \mathrm{~g}$ (99\%) (from isoxazole $3 \mathbf{b}$ ); colorless solid; mp $76-77{ }^{\circ} \mathrm{C}$. ${ }^{1} \mathrm{H}$ NMR $\left(400 \mathrm{MHz}, \mathrm{CDCl}_{3}\right): \delta=7.82-7.74(\mathrm{~m}, 2 \mathrm{H}), 7.48-7.42(\mathrm{~m}, 3 \mathrm{H})$, $5.52(\mathrm{~s}, 1 \mathrm{H}), 4.03(\mathrm{~d}, J=6.6 \mathrm{~Hz}, 2 \mathrm{H}), 2.26-2.10(\mathrm{~m}, 1 \mathrm{H}), 1.07(\mathrm{~d}, J=$ $6.7 \mathrm{~Hz}, 6 \mathrm{H})$.

${ }^{13} \mathrm{C}$ NMR $\left(100 \mathrm{MHz}, \mathrm{CDCl}_{3}\right): \delta=174.0,164.1,129.9,129.7,128.8$, 126.4, 78.4, 75.6, 28.1, 18.8 .

HRMS (ESI): $m / z[\mathrm{M}+\mathrm{Na}]^{+}$calcd for $\mathrm{C}_{13} \mathrm{H}_{15} \mathrm{NNaO}_{2}{ }^{+}: 240.0995$; found: 240.0998 .

\section{Preparation of 3-(3,4-Dimethoxyphenyl)-5-methoxyisoxazole (5m)}

A solution of diazomethane ( $31.5 \mathrm{mmol}$ ) in $\mathrm{Et}_{2} \mathrm{O}$, prepared from $\mathrm{N}$-nitroso- $\mathrm{N}$-methylurea and $\mathrm{KOH}$, was added dropwise at $0{ }^{\circ} \mathrm{C}$ to a stirred suspension of 3-(3,4-dimethoxyphenyl)-5-methoxyisoxazolone $(4 a)^{21 b}(10.5 \mathrm{mmol})$ in anhydrous $\mathrm{Et}_{2} \mathrm{O}(50 \mathrm{~mL})$. The resulting mixture was stirred at ambient temperature for $2 \mathrm{~h}$ and then concentrated under reduced pressure. The residue was purified by column chromatography (silica gel; hexane/EtOAc, 3:1), followed by crystallization from $\mathrm{Et}_{2} \mathrm{O}$-hexane.

Yield: $1.88 \mathrm{~g}$ (76\%); colorless solid; $\mathrm{mp} 69-71{ }^{\circ} \mathrm{C}$.

${ }^{1} \mathrm{H}$ NMR $\left(400 \mathrm{MHz}, \mathrm{CDCl}_{3}\right): \delta=7.42-7.34(\mathrm{~m}, 1 \mathrm{H}), 7.27-7.22(\mathrm{~m}, 1 \mathrm{H})$, 6.96-6.88 (m, $1 \mathrm{H}), 5.50(\mathrm{~s}, 1 \mathrm{H}), 4.05(\mathrm{~s}, 3 \mathrm{H}), 3.95(\mathrm{~s}, 3 \mathrm{H}), 3.93$ (s, $3 \mathrm{H})$.

${ }^{13} \mathrm{C}$ NMR $\left(100 \mathrm{MHz}, \mathrm{CDCl}_{3}\right): \delta=174.3,163.9,150.6,149.2,122.3$, 119.7, 111.0, 108.9, 75.1, 58.8, 56.0, 55.9.

HRMS (ESI): $m / z[\mathrm{M}+\mathrm{Na}]^{+}$calcd for $\mathrm{C}_{12} \mathrm{H}_{13} \mathrm{NNaO}_{4}{ }^{+}: 258.0737$; found: 258.0739 .

\section{Bromoisoxazoles 6 and 6h; General Procedure}

A solution of the isoxazole $\mathbf{5 f}$ or $\mathbf{5 h}(2 \mathrm{mmol})$ and $\mathrm{N}$-bromosuccinimide (392 mg, $2.2 \mathrm{mmol}$ ) in $\mathrm{CHCl}_{3}(25 \mathrm{~mL}$ ) was stirred at ambient temperature for $3 \mathrm{~h}$. The reaction mixture was diluted with $10 \% \mathrm{Na}_{2} \mathrm{~S}_{2} \mathrm{O}_{3}$ $(60 \mathrm{~mL})$, and extracted with $\mathrm{CH}_{2} \mathrm{Cl}_{2}(3 \times 20 \mathrm{~mL})$. The combined organic extracts were dried over anhydrous $\mathrm{Na}_{2} \mathrm{SO}_{4}$, the solvent was removed under reduced pressure, and the product was purified by silica gel flash chromatography (hexane/EtOAc, 5:1), followed by crystallization from $\mathrm{Et}_{2} \mathrm{O}$-hexane.

\section{4-Bromo-3-(2-bromophenyl)-5-methoxyisoxazole (6f)}

Yield: $466 \mathrm{mg}$ (70\%) (from isoxazole 5f); colorless solid; mp 108$109{ }^{\circ} \mathrm{C}$.

${ }^{1} \mathrm{H}$ NMR $\left(400 \mathrm{MHz}, \mathrm{CDCl}_{3}\right): \delta=7.75-7.69(\mathrm{~m}, 1 \mathrm{H}), 7.48-7.34(\mathrm{~m}, 3 \mathrm{H})$, $4.26(\mathrm{~s}, 3 \mathrm{H})$.

${ }^{13} \mathrm{C}$ NMR $\left(100 \mathrm{MHz}, \mathrm{CDCl}_{3}\right): \delta=169.0,164.5,133.1,131.5(2 \mathrm{C}), 129.7$, 127.3, 123.1, 68.9, 58.6.

HRMS (ESI): $m / z[\mathrm{M}+\mathrm{Na}]^{+}$calcd for $\mathrm{C}_{10} \mathrm{H}_{7}{ }^{79} \mathrm{Br}^{81} \mathrm{BrNNaO}_{2}{ }^{+}$: 355.8716 ; found: 355.8718 . 
4-Bromo-3-(2,4-dimethoxyphenyl)-5-methoxyisoxazole (6h)

Yield: $440 \mathrm{mg}$ (70\%) (from isoxazole $\mathbf{5 h}$ ); colorless solid; mp 71-73 ${ }^{\circ} \mathrm{C}$. ${ }^{1} \mathrm{H} \mathrm{NMR}\left(400 \mathrm{MHz}, \mathrm{CDCl}_{3}\right): \delta=7.37-7.31(\mathrm{~m}, 1 \mathrm{H}), 6.62-6.55(\mathrm{~m}, 2 \mathrm{H})$, $4.21(\mathrm{~s}, 3 \mathrm{H}), 3.87(\mathrm{~s}, 3 \mathrm{H}), 3.85(\mathrm{~s}, 3 \mathrm{H})$.

${ }^{13} \mathrm{C}$ NMR $\left(100 \mathrm{MHz}, \mathrm{CDCl}_{3}\right): \delta=168.6,162.9,162.7,158.7,131.7$, $110.0,104.7,98.9,69.4,58.3,55.6,55.4$.

HRMS (ESI): $m / z$ [M + H] $]^{+}$calcd for $\mathrm{C}_{12} \mathrm{H}_{13}{ }^{79} \mathrm{BrNO}_{4}^{+}$: 314.0022 ; found: 314.0027.

\section{Bromoazirines 1c,d,f,h; General Procedure}

A suspension of isoxazole $\mathbf{6 c}, \mathbf{d}, \mathbf{f}, \mathbf{h}(2 \mathrm{mmol})$ and $\mathrm{FeSO}_{4} \cdot 7 \mathrm{H}_{2} \mathrm{O}(239 \mathrm{mg}$, $1.2 \mathrm{mmol}, 60 \mathrm{~mol} \%)$ in $\mathrm{MeCN}(15 \mathrm{~mL})$ was stirred at ambient temperature for $5 \mathrm{~h}$, then the solvent was removed under reduced pressure. In order to remove iron compounds, the product was purified by silica gel flash chromatography (hexane/EtOAc, 10:1), followed by crystallization from $\mathrm{Et}_{2} \mathrm{O}$-hexane.

Methyl 2-Bromo-3-[4-(tert-butyl)phenyl]-2H-azirine-2-carboxylate (1c)

Yield: $608 \mathrm{mg}$ (98\%) (from isoxazole $\mathbf{6} \mathbf{c}^{22}$ ); colorless solid; mp 85$86^{\circ} \mathrm{C}$.

${ }^{1} \mathrm{H}$ NMR $\left(400 \mathrm{MHz}, \mathrm{CDCl}_{3}\right): \delta=7.94-7.88(\mathrm{~m}, 2 \mathrm{H}), 7.70-7.64(\mathrm{~m}, 2 \mathrm{H})$, $3.82(\mathrm{~s}, 3 \mathrm{H}), 1.40(\mathrm{~s}, 9 \mathrm{H})$.

${ }^{13} \mathrm{C}$ NMR $\left(100 \mathrm{MHz}, \mathrm{CDCl}_{3}\right): \delta=167.4,163.8,159.5,130.9,126.8$, 116.7, 54.1, 43.9, 35.6, 31.0.

HRMS (ESI): $m / z[\mathrm{M}+\mathrm{Na}]^{+}$calcd for $\mathrm{C}_{14} \mathrm{H}_{16}{ }^{79} \mathrm{BrNNaO}_{2}{ }^{+}: 332.0257$; found: 332.0259 .

\section{Methyl 2-Bromo-3-(2,4-dimethylphenyl)-2H-azirine-2-carboxyl-} ate (1d)

Yield: $485 \mathrm{mg}$ (86\%) (from isoxazole $\mathbf{6 d}^{23}$ ); colorless solid; mp 60$61{ }^{\circ} \mathrm{C}$.

$\left.{ }^{1} \mathrm{H} \mathrm{NMR} \mathrm{(400} \mathrm{MHz,} \mathrm{CDCl}_{3}\right): \delta=7.75-7.68(\mathrm{~m}, 1 \mathrm{H}), 7.28-7.22(\mathrm{~m}, 2 \mathrm{H})$, $3.83(\mathrm{~s}, 3 \mathrm{H}), 2.67(\mathrm{~s}, 3 \mathrm{H}), 2.46(\mathrm{~s}, 3 \mathrm{H})$.

${ }^{13} \mathrm{C}$ NMR $\left(100 \mathrm{MHz}, \mathrm{CDCl}_{3}\right): \delta=167.6,162.9,146.3,142.6,132.5$, 132.3, 127.6, 115.8, 54.1, 43.5, 21.9, 19.7 .

HRMS (ESI): $m / z[\mathrm{M}+\mathrm{Na}]^{+}$calcd for $\mathrm{C}_{12} \mathrm{H}_{12}{ }^{79} \mathrm{BrNNaO}_{2}{ }^{+}: 303.9944$; found: 303.9948 .

\section{Methyl 2-Bromo-3-(2-bromophenyl)-2H-azirine-2-carboxylate} (1f)

Yield: $619 \mathrm{mg}$ (93\%) (from isoxazole $6 \mathbf{6}$ ); colorless solid; mp $83-84^{\circ} \mathrm{C}$. ${ }^{1} \mathrm{H}$ NMR $\left(400 \mathrm{MHz}, \mathrm{CDCl}_{3}\right): \delta=8.07-8.00(\mathrm{~m}, 1 \mathrm{H}), 7.86-7.79(\mathrm{~m}, 1 \mathrm{H})$, 7.65-7.55 (m, $2 \mathrm{H}), 3.84$ (s, $3 \mathrm{H})$.

${ }^{13} \mathrm{C}$ NMR $\left(100 \mathrm{MHz}, \mathrm{CDCl}_{3}\right): \delta=167.0,164.9,136.0,134.4,133.6$, 128.2, 126.3, 120.8, 54.2, 45.0.

HRMS (ESI): $m / z[\mathrm{M}+\mathrm{Na}]^{+}$calcd for $\mathrm{C}_{10} \mathrm{H}_{7}{ }^{79} \mathrm{Br}^{81} \mathrm{BrNNaO}_{2}^{+}: 355.8716$; found: 355.8734 .

Methyl 2-Bromo-3-(2,4-dimethoxyphenyl)-2H-azirine-2-carboxylate (1h)

Yield: $616 \mathrm{mg}$ (98\%) (from isoxazole $\mathbf{6 h}$ ); colorless solid; $\mathrm{mp} 85-86{ }^{\circ} \mathrm{C}$. ${ }^{1} \mathrm{H} \mathrm{NMR}\left(400 \mathrm{MHz}, \mathrm{CDCl}_{3}\right): \delta=7.72-7.66(\mathrm{~m}, 1 \mathrm{H}), 6.71-6.65(\mathrm{~m}, 1 \mathrm{H})$, 6.59-6.56 (m, $1 \mathrm{H}), 3.99$ (s, $3 \mathrm{H}), 3.93$ (s, $3 \mathrm{H}), 3.80$ (s, $3 \mathrm{H})$.

${ }^{13} \mathrm{C}$ NMR $\left(100 \mathrm{MHz}, \mathrm{CDCl}_{3}\right): \delta=167.9,167.2,162.8,159.6,134.7$, 106.4, 101.4, 98.7, 56.1, 55.9, 53.9, 43.7.
HRMS (ESI): $m / z[\mathrm{M}+\mathrm{Na}]^{+}$calcd for $\mathrm{C}_{12} \mathrm{H}_{12}{ }^{79} \mathrm{BrNNaO}_{4}{ }^{+}$: 335.9842; found: 335.9845 .

\section{Bromoazirines 1i,j,m; General Procedure}

A solution of isoxazole $\mathbf{5 i} \mathbf{j}, \mathbf{m}$ ( $2 \mathrm{mmol}$ ) and $\mathrm{N}$-bromosuccinimide (392 $\mathrm{mg}, 2.2 \mathrm{mmol})$ in $\mathrm{CHCl}_{3}(25 \mathrm{~mL}$ ) was stirred at ambient temperature for $3 \mathrm{~h}$. The reaction mixture was diluted with $10 \% \mathrm{Na}_{2} \mathrm{~S}_{2} \mathrm{O}_{3}(60 \mathrm{~mL})$, and extracted with $\mathrm{CH}_{2} \mathrm{Cl}_{2}(3 \times 20 \mathrm{~mL})$. The combined organic extracts were dried over anhydrous $\mathrm{Na}_{2} \mathrm{SO}_{4}$. The solvent was removed under reduced pressure, and the obtained bromoisoxazole $\mathbf{6 i , j , m}$ was used in the next step without further purification. A suspension of isoxazole $\mathbf{6 i}, \mathbf{j}, \mathbf{m}$ and $\mathrm{FeSO}_{4} \cdot 7 \mathrm{H}_{2} \mathrm{O}(239 \mathrm{mg}, 1.2 \mathrm{mmol}, 60 \mathrm{~mol} \%)$ in $\mathrm{MeCN}(15$ $\mathrm{mL}$ ) was stirred at ambient temperature for $5 \mathrm{~h}$, then the solvent was removed under reduced pressure. In order to remove iron compounds, the product was purified by silica gel flash chromatography (hexane/EtOAc, 10:1), followed by crystallization from $\mathrm{Et}_{2} \mathrm{O}$-hexane.

Isobutyl 2-Bromo-3-phenyl-2H-azirine-2-carboxylate (1i)

Yield: $539 \mathrm{mg}(91 \%)$ (from isoxazole $\mathbf{5 i}$ ); colorless oil.

${ }^{1} \mathrm{H}$ NMR $\left(400 \mathrm{MHz}, \mathrm{CDCl}_{3}\right): \delta=8.02-7.93(\mathrm{~m}, 2 \mathrm{H}), 7.79-7.71(\mathrm{~m}, 1 \mathrm{H})$, 7.69-7.60 (m, $2 \mathrm{H}), 4.08-3.94(\mathrm{~m}, 2 \mathrm{H}), 2.06-1.88(\mathrm{~m}, 1 \mathrm{H}), 0.95-0.86$ $(\mathrm{m}, 6 \mathrm{H})$.

${ }^{13} \mathrm{C}$ NMR $\left(100 \mathrm{MHz}, \mathrm{CDCl}_{3}\right): \delta=166.7,164.6,135.0,130.8,129.6$, $119.8,73.2,44.2,27.6,18.8$.

HRMS (ESI): $m / z[\mathrm{M}+\mathrm{Na}]^{+}$calcd for $\mathrm{C}_{13} \mathrm{H}_{14}{ }^{79} \mathrm{BrNNaO}_{2}{ }^{+}: 318.0100$; found: 318.0093 .

\section{Benzyl 2-Bromo-3-phenyl-2H-azirine-2-carboxylate (1j)}

Yield: $594 \mathrm{mg}(90 \%)$ (from isoxazole $\mathbf{5} \mathbf{j}^{14 \mathrm{a}}$ ); colorless solid; mp 60$61{ }^{\circ} \mathrm{C}$.

${ }^{1} \mathrm{H}$ NMR $\left(400 \mathrm{MHz}, \mathrm{CDCl}_{3}\right): \delta=7.98-7.92(\mathrm{~m}, 2 \mathrm{H}), 7.78-7.72(\mathrm{~m}, 1 \mathrm{H})$, 7.67-7.61 (m, 2 H), 7.39-7.30 (m, 5 H), 5.28 (s, 2 H).

${ }^{13} \mathrm{C} \mathrm{NMR}\left(100 \mathrm{MHz}, \mathrm{CDCl}_{3}\right): \delta=166.6,164.4,135.1,134.9,130.9$, 129.6, 128.5, 128.4, 127.9, 119.6, 68.7, 43.9.

HRMS (ESI): $\mathrm{m} / z[\mathrm{M}+\mathrm{Na}]^{+}$calcd for $\mathrm{C}_{16} \mathrm{H}_{12}{ }^{79} \mathrm{BrNNaO}_{2}{ }^{+}$: 351.9944; found: 351.9949 .

Methyl 2-Bromo-3-(3,4-dimethoxyphenyl)-2H-azirine-2-carboxylate (1m)

Yield: $597 \mathrm{mg}$ (95\%) (from isoxazole $\mathbf{5 m}$ ); colorless solid; mp 102$103^{\circ} \mathrm{C}$.

${ }^{1} \mathrm{H}$ NMR $\left(400 \mathrm{MHz}, \mathrm{CDCl}_{3}\right): \delta=7.61-7.49(\mathrm{~m}, 1 \mathrm{H}), 7.49-7.39(\mathrm{~m}, 1 \mathrm{H})$, 7.13-7.03 (m, $1 \mathrm{H}), 4.01$ (s, $3 \mathrm{H}), 3.98$ (s, $3 \mathrm{H}), 3.82$ (s, $3 \mathrm{H})$.

${ }^{13} \mathrm{C}$ NMR $\left(100 \mathrm{MHz}, \mathrm{CDCl}_{3}\right): \delta=167.4,163.3,154.9,150.0,126.3$, 111.9, 111.7, 111.4, 56.3, 56.2, 54.1, 44.6.

HRMS (ESI): $m / z[\mathrm{M}+\mathrm{Na}]^{+}$calcd for $\mathrm{C}_{12} \mathrm{H}_{12}{ }^{79} \mathrm{BrNNaO}_{4}^{+}: 335.9842$; found: 335.9835 .

\section{2-Fluoro-2H-azirines 2a-1; General Procedure}

A solution of 2-bromo-2H-azirines 1a-1 ( $1 \mathrm{mmol}$ ) and TBAF· $\mathrm{H}_{2} \mathrm{O}$ (363 $\mathrm{mg}, 1.3 \mathrm{mmol})$ in DCM (5 mL) was stirred at ambient temperature for $4 \mathrm{~h}$. The solvent was removed under reduced pressure and the residue was purified by column chromatography on silica gel (hexane-EtOAc mixture) to give fluoroazirines $\mathbf{2 a - 1}$.

Methyl 2-Fluoro-3-phenyl-2H-azirine-2-carboxylate (2a) ${ }^{13}$

Yield: $155 \mathrm{mg}$ (80\%) (from azirine 1a); colorless solid; mp 58-59 ${ }^{\circ} \mathrm{C}$. 
${ }^{1} \mathrm{H}$ NMR $\left(400 \mathrm{MHz}, \mathrm{CDCl}_{3}\right): \delta=8.02-7.94(\mathrm{~m}, 2 \mathrm{H}), 7.77-7.71(\mathrm{~m}, 1 \mathrm{H})$, 7.68-7.61 (m, $2 \mathrm{H}), 3.88$ (s, $3 \mathrm{H})$.

${ }^{13} \mathrm{C}$ NMR $\left(100 \mathrm{MHz}, \mathrm{CDCl}_{3}\right): \delta=167.0$ (d, $\left.J=43.4 \mathrm{~Hz}\right), 166.0$ (d, $J=$ $11.5 \mathrm{~Hz}), 135.0,130.7,129.6,120.2,78.1$ (d, $J=264.6 \mathrm{~Hz}$ ), 53.3.

HRMS (ESI): $m / z$ [M + Na] calcd for $\mathrm{C}_{10} \mathrm{H}_{8} \mathrm{FNNaO}_{2}{ }^{+}: 216.0431$; found: 216.0428.

\section{Methyl 2-Fluoro-3-(p-tolyl)-2H-azirine-2-carboxylate (2b $)^{13}$}

Yield: $170 \mathrm{mg}$ (82\%) (from azirine 1b); colorless solid; mp 44-45 ${ }^{\circ} \mathrm{C}$. ${ }^{1} \mathrm{H}$ NMR $\left(400 \mathrm{MHz}, \mathrm{CDCl}_{3}\right): \delta=7.89-7.83(\mathrm{~m}, 2 \mathrm{H}), 7.47-7.40(\mathrm{~m}, 2 \mathrm{H})$, $3.86(\mathrm{~s}, 3 \mathrm{H}), 2.50(\mathrm{~s}, 3 \mathrm{H})$.

${ }^{13} \mathrm{C}$ NMR $\left(100 \mathrm{MHz}, \mathrm{CDCl}_{3}\right): \delta=167.2(\mathrm{~d}, J=43.9 \mathrm{~Hz}), 165.4(\mathrm{~d}$, $J=11.5 \mathrm{~Hz}$ ), 146.4, 130.8, 130.3, 117.3, 78.1 (d, $J=263.0 \mathrm{~Hz}), 53.3$, 22.0.

HRMS (ESI): $m / z[\mathrm{M}+\mathrm{Na}]^{+}$calcd for $\mathrm{C}_{11} \mathrm{H}_{10} \mathrm{FNNaO}_{2}{ }^{+}: 230.0588$; found: 230.0579 .

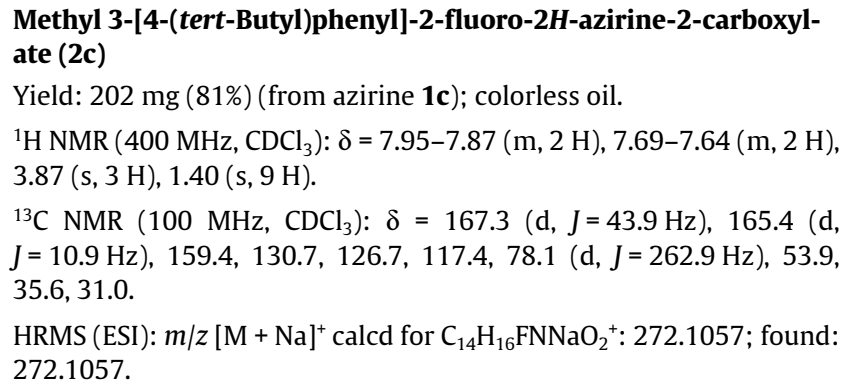

${ }^{13} \mathrm{C}$ NMR (100 MHz, $\left.\mathrm{CDCl}_{3}\right): \delta=167.3(\mathrm{~d}, J=43.9 \mathrm{~Hz}), 165.4(\mathrm{~d}$, $J=10.9 \mathrm{~Hz}$ ), 159.4, 130.7, 126.7, 117.4, 78.1 (d, $J=262.9 \mathrm{~Hz}$ ), 53.9, 35.6, 31.0.

HRMS (ESI): $m / z$ [M + Na] $]^{+}$calcd for $\mathrm{C}_{14} \mathrm{H}_{16} \mathrm{FNNaO}_{2}^{+}: 272.1057$; found: 272.1057.

\section{Methyl 3-(2,4-Dimethylphenyl)-2-fluoro-2H-azirine-2-carboxyl- ate (2d)}

Yield: $190 \mathrm{mg}$ (86\%) (from azirine 1d); colorless solid; mp 64-65 ${ }^{\circ} \mathrm{C}$. ${ }^{1} \mathrm{H}$ NMR $\left(400 \mathrm{MHz}, \mathrm{CDCl}_{3}\right): \delta=7.76-7.69(\mathrm{~m}, 1 \mathrm{H}), 7.27-7.20(\mathrm{~m}, 2 \mathrm{H})$, $3.87(\mathrm{~s}, 3 \mathrm{H}), 2.65(\mathrm{~s}, 3 \mathrm{H}), 2.45(\mathrm{~s}, 3 \mathrm{H})$.

${ }^{13} \mathrm{C}$ NMR (100 MHz, $\mathrm{CDCl}_{3}$ ): $\delta=167.5(\mathrm{~d}, J=44.4 \mathrm{~Hz}$ ), 164.3 (d, $J=11.0 \mathrm{~Hz}), 146.1,142.1,132.21,132.15,127.5,116.2,77.2$ (d, $J=261.9 \mathrm{~Hz}), 53.2,21.9,19.6$.

HRMS (ESI): $m / z$ [M + Na] $]^{+}$calcd for $\mathrm{C}_{12} \mathrm{H}_{12} \mathrm{FNNaO}_{2}^{+}: 244.2168$; found: 244.2169.

\section{Methyl 2-Fluoro-3-(4-nitrophenyl)-2H-azirine-2-carboxylate (2e)} Yield: $155 \mathrm{mg}$ (65\%) (from azirine 1e); colorless solid; mp 115-116 ${ }^{\circ} \mathrm{C}$. $\left.{ }^{1} \mathrm{H} \mathrm{NMR} \mathrm{(400} \mathrm{MHz,} \mathrm{CDCl}_{3}\right): \delta=8.53-8.48(\mathrm{~m}, 2 \mathrm{H}), 8.22-8.16(\mathrm{~m}, 2 \mathrm{H})$, $3.91(\mathrm{~s}, 3 \mathrm{H})$.

${ }^{13} \mathrm{C}$ NMR (100 MHz, $\mathrm{CDCl}_{3}$ ): $\delta=166.3(\mathrm{~d}, J=10.8 \mathrm{~Hz}$ ), 166.1 (d, $J=43.3 \mathrm{~Hz}), 151.4,131.6,125.8,124.7,77.8(\mathrm{~d}, J=268.8 \mathrm{~Hz}), 53.6$.

HRMS (ESI): $m / z$ [M + Na] $]^{+}$calcd for $\mathrm{C}_{10} \mathrm{H}_{7} \mathrm{FN}_{2} \mathrm{NaO}_{4}{ }^{+}: 261.0282$; found: 261.0289 .

\section{Methyl 3-(2-Bromophenyl)-2-fluoro-2H-azirine-2-carboxylate (2f)}

Yield: $204 \mathrm{mg}$ (75\%) (from azirine 1f); colorless solid; mp 45-46 ${ }^{\circ} \mathrm{C}$.

${ }^{1} \mathrm{H} \mathrm{NMR}\left(400 \mathrm{MHz}, \mathrm{CDCl}_{3}\right): \delta=8.13-8.06(\mathrm{~m}, 1 \mathrm{H}), 7.84-7.77(\mathrm{~m}, 1 \mathrm{H})$, 7.63-7.55 (m, $2 \mathrm{H}), 3.89$ (s, $3 \mathrm{H})$.

${ }^{13} \mathrm{C}$ NMR (100 MHz, $\left.\mathrm{CDCl}_{3}\right): \delta=166.74(\mathrm{~d}, J=12.2 \mathrm{~Hz}), 166.73$ (d, $J=43.2 \mathrm{~Hz}), \quad 136.0,134.1,132.8,128.2,126.5,121.3,78.2$ (d, $J=266.5 \mathrm{~Hz}), 53.4$.
HRMS (ESI): $m / z[\mathrm{M}+\mathrm{Na}]^{+}$calcd for $\mathrm{C}_{10} \mathrm{H}_{7}{ }^{79} \mathrm{BrFNNaO}_{2}^{+}$: 293.9536; found: 293.9529 .

Methyl 2-Fluoro-3-(4-methoxyphenyl)-2H-azirine-2-carboxylate (2g)

Yield: $181 \mathrm{mg}$ (81\%) (from azirine 1g); colorless solid; mp 53-54 ${ }^{\circ} \mathrm{C}$. ${ }^{1} \mathrm{H} \mathrm{NMR}\left(400 \mathrm{MHz}, \mathrm{CDCl}_{3}\right): \delta=7.95-7.87(\mathrm{~m}, 2 \mathrm{H}), 7.15-7.08(\mathrm{~m}, 2 \mathrm{H})$, $3.94(\mathrm{~s}, 3 \mathrm{H}), 3.86(\mathrm{~s}, 3 \mathrm{H})$.

${ }^{13} \mathrm{C}$ NMR $\left(100 \mathrm{MHz}, \mathrm{CDCl}_{3}\right): \delta=167.4(\mathrm{~d}, J=44.7 \mathrm{~Hz}), 165.0,164.3(\mathrm{~d}$, $J=10.9 \mathrm{~Hz}), 133.0,115.2,112.3,78.2(\mathrm{~d}, J=262.7 \mathrm{~Hz}), 55.7,53.2$.

HRMS (ESI): $m / z$ [M + Na] $]^{+}$calcd for $\mathrm{C}_{11} \mathrm{H}_{10} \mathrm{FNNaO}_{3}{ }^{+}: 246.0537$; found: 246.0539 .

Methyl 3-(2,4-Dimethoxyphenyl)-2-fluoro-2H-azirine-2-carboxylate (2h)

Yield: $192 \mathrm{mg}$ (76\%) (from azirine 1h); colorless solid; mp 120$121{ }^{\circ} \mathrm{C}$.

${ }^{1} \mathrm{H} \mathrm{NMR}\left(400 \mathrm{MHz}, \mathrm{CDCl}_{3}\right): \delta=7.57-7.51(\mathrm{~m}, 1 \mathrm{H}), 7.49-7.43(\mathrm{~m}, 1 \mathrm{H})$, 7.09-7.03 (m, $1 \mathrm{H}), 4.00(\mathrm{~s}, 3 \mathrm{H}), 3.98(\mathrm{~s}, 3 \mathrm{H}), 3.87(\mathrm{~s}, 3 \mathrm{H})$.

${ }^{13} \mathrm{C}$ NMR (100 MHz, $\left.\mathrm{CDCl}_{3}\right): \delta=167.3(\mathrm{~d}, J=44.5 \mathrm{~Hz}), 164.4(\mathrm{~d}$, $J=11.3 \mathrm{~Hz}), 154.8,149.9,126.1,112.3,111.8,111.3,78.4$ (d, $J=263.0 \mathrm{~Hz}), 56.3,56.2,53.3$.

HRMS (ESI): $m / z$ [M + Na] $]^{+}$calcd for $\mathrm{C}_{12} \mathrm{H}_{12} \mathrm{FNNaO}_{4}{ }^{+}: 276.0643$; found: 276.0651 .

Isobutyl 2-Fluoro-3-phenyl-2H-azirine-2-carboxylate (2i)

Yield: $195 \mathrm{mg}$ (83\%) (from azirine 1i); colorless oil.

${ }^{1} \mathrm{H}$ NMR $\left(400 \mathrm{MHz}, \mathrm{CDCl}_{3}\right): \delta=8.01-7.94(\mathrm{~m}, 2 \mathrm{H}), 7.77-7.70(\mathrm{~m}, 1 \mathrm{H})$, 7.68-7.62 (m, 2 H), 4.11-4.01 (m, 2 H), 2.06-1.93 (m, 1 H), 0.95-0.90 (m, $6 \mathrm{H})$.

${ }^{13} \mathrm{C}$ NMR (100 MHz, $\left.\mathrm{CDCl}_{3}\right): \delta=166.6(\mathrm{~d}, J=43.2 \mathrm{~Hz}), 166.2(\mathrm{~d}$, $J=11.5 \mathrm{~Hz}$ ), 134.9, 130.7, 129.6, 120.4, 78.1 (d, $J=264.5 \mathrm{~Hz}), 72.4$, 27.6, 18.8 .

HRMS (ESI): $m / z[\mathrm{M}+\mathrm{Na}]^{+}$calcd for $\mathrm{C}_{13} \mathrm{H}_{14} \mathrm{FNNaO}_{2}^{+}: 258.0901$; found: 258.0907.

\section{Benzyl 2-Fluoro-3-phenyl-2H-azirine-2-carboxylate (2j)}

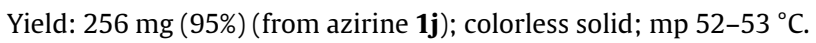
${ }^{1} \mathrm{H}$ NMR (400 MHz, $\left.\mathrm{CDCl}_{3}\right): \delta=7.99-7.93(\mathrm{~m}, 2 \mathrm{H}), 7.77-7.71(\mathrm{~m}, 1 \mathrm{H})$, 7.67-7.61 (m, $2 \mathrm{H}), 7.41-7.35$ (m, $5 \mathrm{H}), 5.32(\mathrm{~s}, 2 \mathrm{H})$.

${ }^{13} \mathrm{C}$ NMR (100 MHz, $\left.\mathrm{CDCl}_{3}\right): \delta=166.6(\mathrm{~d}, J=43.2 \mathrm{~Hz}), 166.1(\mathrm{~d}$, $J=11.2 \mathrm{~Hz}), 135.0,134.8,130.8,129.6,128.7,128.6,128.3,120.9,78.2$ $(\mathrm{d}, J=265.1 \mathrm{~Hz}), 68.1$.

HRMS (ESI): $m / z[\mathrm{M}+\mathrm{Na}]^{+}$calcd for $\mathrm{C}_{16} \mathrm{H}_{12} \mathrm{FNNaO}_{2}{ }^{+}: 292.0744$; found: 292.0733 .

\section{(2-Fluoro-3-phenyl-2H-azirin-2-yl)(pyrrolidin-1-yl)methanone} (2k)

Yield: $149 \mathrm{mg}$ (64\%) (from azirine 1k); colorless solid; $\mathrm{mp} 67-68{ }^{\circ} \mathrm{C}$. ${ }^{1} \mathrm{H}$ NMR $\left(400 \mathrm{MHz}, \mathrm{CDCl}_{3}\right): \delta=8.05-7.98(\mathrm{~m}, 2 \mathrm{H}), 7.72-7.66(\mathrm{~m}, 1 \mathrm{H})$, 7.63-7.58 (m, $2 \mathrm{H}), 4.11-4.01(\mathrm{~m}, 1 \mathrm{H}), 3.94-3.84(\mathrm{~m}, 1 \mathrm{H}), 3.63-3.56$ ( $\mathrm{m}, 2 \mathrm{H}), 2.12-1.87$ ( $\mathrm{m}, 4 \mathrm{H})$.

${ }^{13} \mathrm{C}$ NMR (100 MHz, $\left.\mathrm{CDCl}_{3}\right): \delta=169.7(\mathrm{~d}, J=12.9 \mathrm{~Hz}), 163.3(\mathrm{~d}$, $J=39.3 \mathrm{~Hz}), 134.5,130.7,129.3,121.3,82.3(\mathrm{~d}, J=274.9 \mathrm{~Hz}), 47.1$ (d, $J=9.2 \mathrm{~Hz}), 47.0,26.3(\mathrm{~d}, J=3.2 \mathrm{~Hz}), 23.7$. 
HRMS (ESI): $m / z[\mathrm{M}+\mathrm{H}]^{+}$calcd for $\mathrm{C}_{13} \mathrm{H}_{14} \mathrm{FN}_{2} \mathrm{O}^{+}:$233.1085; found: 233.1086 .

(2-Fluoro-3-phenyl-2H-azirin-2-yl)(morpholino)methanone (21) Yield: $99 \mathrm{mg}$ (40\%) (from azirine 11); colorless solid; mp 53-54 ${ }^{\circ} \mathrm{C}$. ${ }^{1} \mathrm{H} \mathrm{NMR}\left(400 \mathrm{MHz}, \mathrm{CDCl}_{3}\right): \delta=8.11-8.02(\mathrm{~m}, 2 \mathrm{H}), 7.77-7.69(\mathrm{~m}, 1 \mathrm{H})$, 7.68-7.60 (m, 2 H), 4.14-4.01 (m, 2 H), 3.90-3.65 (m, $6 \mathrm{H})$.

${ }^{13} \mathrm{C}$ NMR (100 MHz, $\left.\mathrm{CDCl}_{3}\right): \delta=171.0(\mathrm{~d}, J=13.0 \mathrm{~Hz}), 163.1$ (d, $J=39.3 \mathrm{~Hz}$ ), 134.9, 130.8, 129.5, 121.1, 82.2 (d, $J=274.2 \mathrm{~Hz}), 67.1$, $66.4,46.5,42.9$.

HRMS (ESI): $m / z[\mathrm{M}+\mathrm{Na}]^{+}$calcd for $\mathrm{C}_{13} \mathrm{H}_{13} \mathrm{FN}_{2} \mathrm{NaO}_{2}^{+}$: 271.0853; found: 271.0855 .

\section{Gram-Scale Synthesis of Fluoroazirine 2a from Bromoazirine 1a}

A solution of azirine $1 \mathbf{a}(2.2 \mathrm{~g}, 8.66 \mathrm{mmol})$ and $\mathrm{Bu}_{4} \mathrm{NF} \cdot \mathrm{H}_{2} \mathrm{O}(3.14 \mathrm{~g}$, $11.26 \mathrm{mmol})$ in DCM $(40 \mathrm{~mL})$ was stirred at ambient temperature for $4 \mathrm{~h}$. The solvent was removed under reduced pressure and the residue was purified by column chromatography on silica gel (hexane/EtOAc mixture) to give methyl 2-fluoro-3-phenyl-2H-azirine-2-carboxylate 2a $(1.34 \mathrm{~g}, 80 \%)$.

\section{2-Iodo-2H-azirines 7a-f; General Procedure}

A solution of 2-bromo-2H-azirines 1a,b,e,j,m,n (1 mmol) and $\mathrm{KI}$ (332 $\mathrm{mg}, 2 \mathrm{mmol}$ ) in acetone ( $5 \mathrm{~mL}$ ) was stirred at ambient temperature for the time indicated below. The solvent was removed under reduced pressure and the residue was purified by column chromatography on silica gel (hexane/EtOAc mixture) and crystallization from $\mathrm{Et}_{2} \mathrm{O}$-hexane to give iodoazirines $\mathbf{7 a - f}$.

\section{Methyl 2-Iodo-3-phenyl-2H-azirine-2-carboxylate (7a) $)^{14 a}$}

Yield: $271 \mathrm{mg}(90 \%)$ (from azirine 1a, $30 \mathrm{~h}$ ); colorless solid; mp 87$88^{\circ} \mathrm{C}$ (lit. $\left.{ }^{14 a} 87-88^{\circ} \mathrm{C}\right)$.

\section{Methyl 2-Iodo-3-(p-tolyl)-2H-azirine-2-carboxylate (7b)}

Yield: $290 \mathrm{mg}$ (92\%) (from azirine 1b, 48 h); colorless solid; mp 81$82{ }^{\circ} \mathrm{C}$.

${ }^{1} \mathrm{H}$ NMR $\left(400 \mathrm{MHz}, \mathrm{CDCl}_{3}\right): \delta=7.89-7.81(\mathrm{~m}, 2 \mathrm{H}), 7.50-7.43(\mathrm{~m}, 2 \mathrm{H})$, $3.80(\mathrm{~s}, 3 \mathrm{H}), 2.51(\mathrm{~s}, 3 \mathrm{H})$.

${ }^{13} \mathrm{C}$ NMR $\left(100 \mathrm{MHz}, \mathrm{CDCl}_{3}\right): \delta=166.5,164.8,146.5,130.9,130.5$, 117.2, 54.3, 22.1, 14.0.

HRMS (ESI): $m / z[\mathrm{M}+\mathrm{Na}]^{+}$calcd for $\mathrm{C}_{11} \mathrm{H}_{10} \mathrm{INNaO}_{2}{ }^{+}: 337.9648$; found: 337.9643.

\section{Methyl 2-Iodo-3-(4-nitrophenyl)-2H-azirine-2-carboxylate (7c)}

Yield: $277 \mathrm{mg}$ (80\%) (from azirine 1e, $40 \mathrm{~h}$ ); colorless solid; mp 131$133^{\circ} \mathrm{C}$.

${ }^{1} \mathrm{H}$ NMR $\left(400 \mathrm{MHz}, \mathrm{CDCl}_{3}\right): \delta=8.58-8.42(\mathrm{~m}, 2 \mathrm{H}), 8.26-8.10(\mathrm{~m}, 2 \mathrm{H})$, $3.84(\mathrm{~s}, 3 \mathrm{H})$.

${ }^{13} \mathrm{C}$ NMR $\left(100 \mathrm{MHz}, \mathrm{CDCl}_{3}\right): \delta=165.7,165.3,151.3,131.6,125.8$, 124.8, 54.6, 12.2 .

HRMS (ESI): $m / z$ [M + Na] $]^{+}$calcd for $\mathrm{C}_{10} \mathrm{H}_{7} \mathrm{IN}_{2} \mathrm{NaO}_{4}{ }^{+}: 368.9343$; found: 368.9348 .
Methyl 3-(3,4-Dimethoxyphenyl)-2-iodo-2H-azirine-2-carboxylate $(7 \mathrm{~d})$

Yield: $325 \mathrm{mg}$ (90\%) [from azirine 1m, KI (2.5 g, 15 mmol), $120 \mathrm{~h}$ ]; colorless solid; $\mathrm{mp} 93-94^{\circ} \mathrm{C}$.

${ }^{1} \mathrm{H}$ NMR $\left(400 \mathrm{MHz}, \mathrm{CDCl}_{3}\right): \delta=7.58-7.49(\mathrm{~m}, 1 \mathrm{H}), 7.46-7.40(\mathrm{~m}, 1 \mathrm{H})$, 7.12-7.03 (m, $1 \mathrm{H}), 4.01$ (s, $3 \mathrm{H}), 3.99$ (s, $3 \mathrm{H}), 3.81$ (s, $3 \mathrm{H})$.

${ }^{13} \mathrm{C}$ NMR $\left(100 \mathrm{MHz}, \mathrm{CDCl}_{3}\right): \delta=166.6,164.2,154.9,150.1,126.2$, $112.1,111.8,111.4,56.3,56.2,54.3,14.9$.

HRMS (ESI): $m / z[\mathrm{M}+\mathrm{Na}]^{+}$calcd for $\mathrm{C}_{12} \mathrm{H}_{12} \mathrm{INNaO}_{4}{ }^{+}: 383.9703$; found: 383.9686 .

Methyl 3-(2,3-Dihydrobenzo[b][1,4]dioxin-6-yl)-2-iodo-2H-azirine-2-carboxylate (7e)

Yield: $323 \mathrm{mg}$ (90\%) [from azirine 1n, KI (2.5 g, $15 \mathrm{mmol}), 120 \mathrm{~h}$ ]; colorless solid; mp $109-110{ }^{\circ} \mathrm{C}$ (dec.).

${ }^{1} \mathrm{H} \mathrm{NMR}\left(400 \mathrm{MHz}, \mathrm{CDCl}_{3}\right): \delta=7.54-7.42(\mathrm{~m}, 2 \mathrm{H}), 7.15-7.05(\mathrm{~m}, 1 \mathrm{H})$, 4.42-4.33 (m, $4 \mathrm{H}), 3.80$ (s, $3 \mathrm{H})$.

${ }^{13} \mathrm{C}$ NMR $\left(100 \mathrm{MHz}, \mathrm{CDCl}_{3}\right): \delta=166.5,164.1,149.7,144.4,125.1$, $119.9,118.8,112.7,64.8,64.0,54.3,14.6$.

HRMS (ESI): $m / z[\mathrm{M}+\mathrm{Na}]^{+}$calcd for $\mathrm{C}_{12} \mathrm{H}_{10} \mathrm{INNaO}_{4}{ }^{+}: 381.9547$; found: 381.9547.

Benzyl 2-Iodo-3-phenyl-2H-azirine-2-carboxylate (7f) Yield: $373 \mathrm{mg}$ (99\%) (from azirine $\mathbf{1 j}$, $48 \mathrm{~h}$ ); colorless oil.

${ }^{1} \mathrm{H}$ NMR (400 MHz, $\left.\mathrm{CDCl}_{3}\right): \delta=8.00-7.90(\mathrm{~m}, 2 \mathrm{H}), 7.80-7.72(\mathrm{~m}, 1 \mathrm{H})$, 7.70-7.61 (m, 2 H), 7.41-7.29 (m, 5 H), 5.25 (s, 2 H).

${ }^{13} \mathrm{C}$ NMR $\left(100 \mathrm{MHz}, \mathrm{CDCl}_{3}\right): \delta=165.8,165.3,135.0$ (2C), 130.9, 129.7, 128.5, 128.4, 127.9, 120.1, 69.0, 13.8 .

HRMS (ESI): $m / z$ [M + Na] $]^{+}$calcd for $\mathrm{C}_{16} \mathrm{H}_{12} \mathrm{INNaO}_{2}{ }^{+}: 399.9805$; found: 399.9800 .

\section{Synthesis of Bromoazirine 1a from 2-Iodoazirine 7a}

A solution of iodoazirine $7 \mathbf{a}(301 \mathrm{mg}, 1 \mathrm{mmol})$ and $\mathrm{Bu}_{4} \mathrm{NBr}(645 \mathrm{mg}$, $2.0 \mathrm{mmol}$ ) in DCM (5 mL) was stirred at ambient temperature for 15 $\mathrm{h}$. The solvent was removed under reduced pressure and the residue was purified by column chromatography on silica gel (hexane/EtOAc, $4: 1)$ to give bromoazirine $\mathbf{1 a}(252 \mathrm{mg}, 99 \%)$.

\section{Synthesis of Chloroazirine 8a from Bromoazirine 1a}

A solution of bromoazirine $\mathbf{1 a}(254 \mathrm{mg}, 1 \mathrm{mmol})$ and $\mathrm{Bu}_{4} \mathrm{NCl}(417 \mathrm{mg}$, $1.5 \mathrm{mmol}$ ) in DCM ( $5 \mathrm{~mL}$ ) was stirred at ambient temperature for 12 $\mathrm{h}$. The solvent was removed under reduced pressure and the residue was purified by column chromatography on silica gel (hexane/EtOAc, 4:1) to give chloroazirine $\mathbf{8 a}$ ( $205 \mathrm{mg}, 98 \%$ ).

\section{Synthesis of Chloroazirine 8a from 2-Iodoazirine 7a}

A solution of iodoazirine $7 \mathbf{a}(301 \mathrm{mg}, 1 \mathrm{mmol})$ and $\mathrm{Bu}_{4} \mathrm{NCl}(249 \mathrm{mg}$, $1.5 \mathrm{mmol}$ ) in DCM (5 mL) was stirred at ambient temperature for $6 \mathrm{~h}$. The solvent was removed under reduced pressure and the residue was purified by column chromatography on silica gel (hexane/EtOAc, 4:1) to give chloroazirine $\mathbf{8 a}$ (205 $\mathrm{mg}, 98 \%$ ).

\section{Funding Information}

We gratefully acknowledge the financial support of the Russian Science Foundation (Grant No. 17-13-01078). 


\section{Acknowledgment}

This research used resources of the 'Magnetic Resonance Research Centre', the 'Chemical Analysis and Materials Research Centre', and the 'Chemistry Educational Centre' of the Research Park of St. Petersburg State University.

\section{Supporting Information}

Supporting information for this article is available online at https://doi.org/10.1055/s-0039-1690200.

\section{References}

(1) Groult, H.; Leroux, F.; Tressaud, A. Modern Synthesis Processes and Reactivity of Fluorinated Compounds, 1st ed; Elsevier: Amsterdam, 2016.

(2) Dohi, T.; Kita, Y. In Iodine Chemistry and Applications; Kaiho, T., Ed.; Wiley: Hoboken, 2014, 303.

(3) Berger, R.; Resnati, G.; Metrangolo, P.; Weberd, E.; Hulliger, J. Chem. Soc. Rev. 2011, 40, 3496.

(4) (a) Zhou, Y.; Wang, J.; Gu, Z.; Wang, S.; Zhu, W.; Aceña, J. L.; Soloshonok, V. A.; Izawa, K.; Liu, H. Chem. Rev. 2016, 116, 422. (b) Barnes-Seeman, D.; Beck, J.; Springer, C. Curr. Top. Med. Chem. 2014, 14, 855.

(5) Fluorine and Health: Molecular Imaging, Biomedical Materials and Pharmaceuticals; Tressaud, A.; Haufe, G., Ed.; Elsevier: Amsterdam, 2008.

(6) Ametamey, S. M.; Honer, M.; Schubiger, P. A. Chem. Rev. 2008 , $108,1501$.

(7) (a) Chacko, A. M.; Divgi, C. R. Med. Chem. 2011, 7, 395. (b) Koehler, L.; Gagnon, K.; McQuarrie, S.; Wuest, F. Molecules 2010, 15, 2686.

(8) For recent reviews on $2 \mathrm{H}$-azirine chemistry, see: (a) Khlebnikov, A. F.; Novikov, M. S.; Rostovskii, N. V. Tetrahedron 2019, 75, 2555. (b) Zhou, H.; Shen, M.-H.; Xu, H.-D. Synlett 2016, 27, 2171. (c) Khlebnikov, A. F.; Novikov, M. S. Top. Heterocycl. Chem. 2015, 41, 143. (d) Huang, C.-Y.; Doyle, A. G. Chem. Rev. 2014, 114, 8153. (e) Khlebnikov, A. F.; Novikov, M. S. Tetrahedron 2013, 69, 3363.
(9) Smetanin, I. A.; Novikov, M. S.; Rostovskii, N. V.; Khlebnikov, A. F.; Starova, G. L.; Yufit, D. S. Tetrahedron 2015, 71, 4616.

(10) Rostovskii, N. V.; Smetanin, I. A.; Agafonova, A. V.; Sakharov, P. A.; Ruvinskaya, J. O.; Khlebnikov, A. F.; Novikov, M. S. Org. Biomol. Chem. 2018, 16, 3248.

(11) (a) Alonso-Cruz, C. R.; Kennedy, A. R.; Rodríguez, M. S.; Suárez, E. J. Org. Chem. 2008, 73, 4116. (b) Alonso-Cruz, C. R.; Kennedy, A. R.; Rodríguez, M. S.; Suárez, E. Tetrahedron Lett. 2007, 48, 7207.

(12) Pinho e Melo, T. M. V. D.; Lopes, C. S. J.; Cardoso, A. L.; Rocha Gonsalves, A. M. d'A. Tetrahedron 2001, 57, 6203.

(13) Zhang, Y.; Zhao, X.; Zhuang, C.; Wang, S.; Zhang-Negrerie, D.; Du, Y. Adv. Synth. Catal. 2018, 360, 2107.

(14) (a) Rostovskii, N. V.; Agafonova, A. V.; Smetanin, I. A.; Novikov, M. S.; Ruvinskaya, J. O.; Starova, G. L. Khlebnikov A. F. Synthesis 2017, 49, 4478. (b) Agafonova, A. V.; Smetanin, I. A.; Rostovskii, N. V.; Khlebnikov, A. F.; Novikov, M. S. Chem. Heterocycl. Compd. 2017, 53, 1068.

(15) Okamoto, K.; Nanya, A.; Eguchi, A.; Ohe, K. Angew. Chem. Int. Ed. 2018, 57, 1039.

(16) (a) Liang, T.; Neumann, C. N.; Ritter, T. Angew. Chem. Int. Ed. 2013, 52, 8214. (b) Langlois, B.; Gilbert, L.; Forat, G. Ind. Chem. Libr. 1996, 8, 244

(17) Worrell, B. T.; Hein, J. E.; Fokin, V. V. Angew. Chem. Int. Ed. 2012, $51,11791$.

(18) Allen, L. J.; Muhuhi, J. M.; Bland, D. C.; Merzel, R.; Sanford, M. S. J. Org. Chem. 2014, 79, 5827.

(19) Agafonova, A. V.; Rostovskii, N. V.; Smetanin, I. A.; Starova, G. L.; Khlebnikov, A. F.; Novikov, M. S. J. Org. Chem. 2018, 83, 13473.

(20) Bounioux, C.; Bar-Hen, A.; Yerushalmi-Rozen, R. Chem. Commun. 2015, 51, 6343.

(21) (a) Nishiwaki, T.; Kitamura, T.; Nakano, A. Tetrahedron 1970, 55, 453. (b) Clark, A. D.; Janowski, W. K.; Prager, R. H. Tetrahedron 1999, 55, 3637.

(22) Golubev, A. A.; Smetanin, I. A.; Agafonova, A. V.; Rostovskii, N. V.; Khlebnikov, A. F.; Starova, G. L.; Novikov, M. S. Org. Biomol. Chem. 2019, 17, 6821.

(23) Smetanin, I. A.; Novikov, M. S.; Agafonova, A. V.; Rostovskii, N. V.; Khlebnikov, A. F.; Kudryavtsev, I. V.; Terpilowski, M. A.; Serebriakova, M. K.; Trulioff, A. S.; Goncharov, N. V. Org. Biomol. Chem. 2016, 14, 4479. 